\title{
Lessons Learned from the Early Stage of Convid-19 Pandemic: Clinical Analysis of 17 Related Deaths Associated with the 2019 Novel Coronavirus
}

Cheng Zhang

Jiangsu Province Hospital of Chinese Medicine

Dandan Hong

Jiangsu Province Hospital of Chinese Medicine

Wenyue Chen

Jiangsu Province Hospital of Chinese Medicine

Qun Guan ( $\square$ zhangcheng20171@outlook.com )

Qingling Ren

Research

Keywords: 2019-nCoV, pneumovirus, Wuhan, death

Posted Date: June 22nd, 2020

DOI: https://doi.org/10.21203/rs.3.rs-36607/v1

License: (c) (i) This work is licensed under a Creative Commons Attribution 4.0 International License.

Read Full License 


\section{Abstract}

Background Since the end of December 2019, a pneumonia outbreak has occurred in Wuhan, Hubei Province, China. An epidemiological survey showed that the pneumovirus infection was closely related to the Huanan Seafood Wholesale Market in Wuhan, causing great panic among the general population. Through isolation of the pneumovirus strain and whole-genome sequencing, an infection model of the pneumovirus in human airway epithelial cells was established, and the pneumovirus was identified as the 2019 novel coronavirus (2019-nCoV), which belongs to the $\beta$-coronavirus genus. This study focused on the different characteristics of 2019-nCoV, which we learned at the early stage of Covid-19 pandemic will help us to save more lives during the outbreak.

Methods The data of 17 deaths from 13 hospitals in Wuhan were obtained, and the initial symptoms, clinical features during the progression of the disease, and treatment processes were well analyzed.

Results At the initial phase of the outbreak of 2019-nCoV, the lack of effective drugs for pneumonia has led to lethal results in people who are often elderly with multiple underlying conditions. Males were affected more than females. Fever, dry coughing, shortness of breath were the primary presentations, but not all. Chest images showed typical features of viral pneumonia. Respiratory support treatment were needed, although sometimes did not improve respiratory distress. All the patients died of multiple organ failure caused by respiratory failure. Many treatments of covid-19 were done in ICU and lasted for a long time, the occupation of public medical resources will put pressure on the present medical system.

Conclusions COVID-19 is different from pneumonia caused by previous coronaviruses, it is more infectious and hard to manage. The initial death population were mainly elderly patients with underlying diseases. Protecting the elderly is an effective method to reduce the number of deaths. In the absence of nucleic acid detection reagents, relying on identifying typical symptoms and making full use of CT can increase the diagnosis of suspicious people. The condition of patients may deteriorate rapidly which increased the complexity of treatment. The increasing number of patients will put great pressure on the existing medical system.

\section{Background:}

Since the end of December 2019, a pneumonia outbreak has occurred in Wuhan, Hubei Province, China. An epidemiological survey showed that the pneumovirus infection was closely related to the Huanan Seafood Wholesale Market in Wuhan, causing great panic among the general population. Through the isolation and whole-genome sequencing of the pneumovirus, an infection model of the pneumovirus in human airway epithelial cells was established, and the pneumovirus was identified as the 2019 novel coronavirus (2019-nCoV), which belongs to the $\beta$-coronavirus genus. The most substantial task for clinicians is to further clarify the clinical pattern of the viral infection. In this study, we performed a clinical analysis of deaths caused by this novel coronavirus-infection pneumonia (NCIP) in the early period of the pneumonia outbreak to clarify the clinical features of the occurrence and development of 
NCIP, the distribution characteristics of deaths caused by NCIP, and the differences from deaths caused by other coronaviruses, which should help to better understand this novel virus and help other countries and regions prevent and treat it.

\section{Methods:}

The data of 17 deaths from 13 hospitals in Wuhan were obtained, and the initial symptoms, clinical features during the progression of the disease, and treatment processes were analyzed.

\section{Results:}

Among the 17 patients, 5 were from Wuhan Jinyintan Hospital (severe patients were transferred from other local hospitals), and the other 12 were from 7 hospitals in Wuhan. At disease onset, the age of the patients ranged from 48 to 89 years old, and the average age was 73 years old $\rrbracket$ most suffered were male. Two patients were younger than 60 years old (48 years old and 53 years old), accounting for $11.8 \%$; 5 patients were between 60 and 70 years old, accounting for $29.8 \%$; 2 patients were between 70 and 80 years old, accounting for $11.8 \%$; and 8 patients were over 80 years old, accounting for $47.1 \%$. Among the 17 patients, 13 were males (76.5\%), and 4 were females (23.5\%). The average age of females was 70 years old, and that of males was 73 years old. Among them, 10 patients had clearly documented underlying diseases, including hypertension (7 cases), diabetes (5 cases), cerebral infarction ( $\mathrm{Cl}, 3$ cases), coronary heart disease (CHD, 2 cases), Parkinson's disease (PD, 2 cases), and liver cirrhosis (LC, 1 case). Of the 10 patients, 2 had underlying pulmonary diseases, including chronic bronchitis (CB) and chronic obstructive pulmonary disease (COPD). The remaining 7 patients did not have a clear record of underlying diseases, and some patients were unable to provide their medical history due to rapid progression of the disease.

Disease onset for 10 patients occurred from late December 2019 to early January 2020 (58.8\%). The earliest onset was December 10, 2019, and the latest onset was January 15,2020 . The most deaths occurred around January 20, 2020 (16/17, 94.1\%).

The main initial onset symptom was fever (11/17), including 1 case of persistent high fever and 6 cases without fever. The accompanying symptoms of fever included 1. cough (9/17), with a dry cough and less paralysis as the main manifestations; 2 . headache and fatigue (4/17), and 3 . chest tightness and shortness of breath, which was aggravated after activity. Some patients without fever had dyspnea, lethargy and other hypoxic conditions when admitted to the hospital. Only 1 patient had vomiting, and no diarrhea occurred (Table 2).

As the disease progressed, all patients had respiratory distress and varying degrees of respiratory failure. Except for 3 older patients whose family refused rescue treatment, the remaining patients underwent invasive endotracheal intubation and assisted mechanical ventilation. Additionally, symptomatic support treatment was performed. Some patients with multisystem underlying diseases developed septic shock, 
liver insufficiency, renal insufficiency, persistent hypoxemia and persistent high fever and were given corresponding support treatment. One patient received extracorporeal membrane oxygenation (ECMO) treatment, but no improvement was observed. One patient developed myocardial enzyme increase. One patient experienced a decrease in muscle strength in $1 \mathrm{limb}$. A total of 12 patients underwent chest imaging, and all results showed pneumonia in the bilateral lungs, with partial patchy and ground-glass like lesions. In severe cases, extensive pulmonary consolidation occurred, but pleural effusion was rare. The disease worsened as symptomatic supportive treatments were not able to improve the pulmonary infection, respiratory failure and hypoxemia, and persistent hypoxia in tissues and organs induced multiple organ dysfunction. The average time from disease onset to death was 16 days; the longest duration was 41 days for the youngest woman (48 years old). With aggravated disease, death occurred after an average of 3.4 days.

Among the 17 patients, 2 patients received a nucleic acid test for respiratory specimens, with positive results; the remaining patients did not receive etiological examinations. The diagnosis of pneumonia was primarily based on the clinical symptoms and chest imaging results. Some patients were critically ill and were unable to undergo pathological and imaging examinations in a timely manner. 
Table 1: Basic information of the 17 patients

Number of cases

(cases)

Age (years)

Mean age

73

Age distribution

48-89

40-50

$1(5.9 \%)$

$50-60$

$1(5.9 \%)$

$60-70$

$5(29.4 \%)$

$70-80$

$2(11.8 \%)$

$80-90$

$8(47.1 \%)$

Sex

Male

$13(76.5 \%)$

Female

$4(23.5 \%)$

Underlying diseases

Clear medical records

(combination)

Hypertension

7

Diabetes

5

$\mathrm{Cl}$

3

PD

2

CHD

2

Chronic renal insufficiency

(CRF)

$\begin{array}{ll}\text { COPD } & 1 \\ \text { CB } & 1 \\ \text { LC } & 1\end{array}$

No medical records

7 (41.2\%)

Disease outcome

Death

$17(100 \%)$ 
Table 2: Initial symptoms of and treatment process for the 17 patients

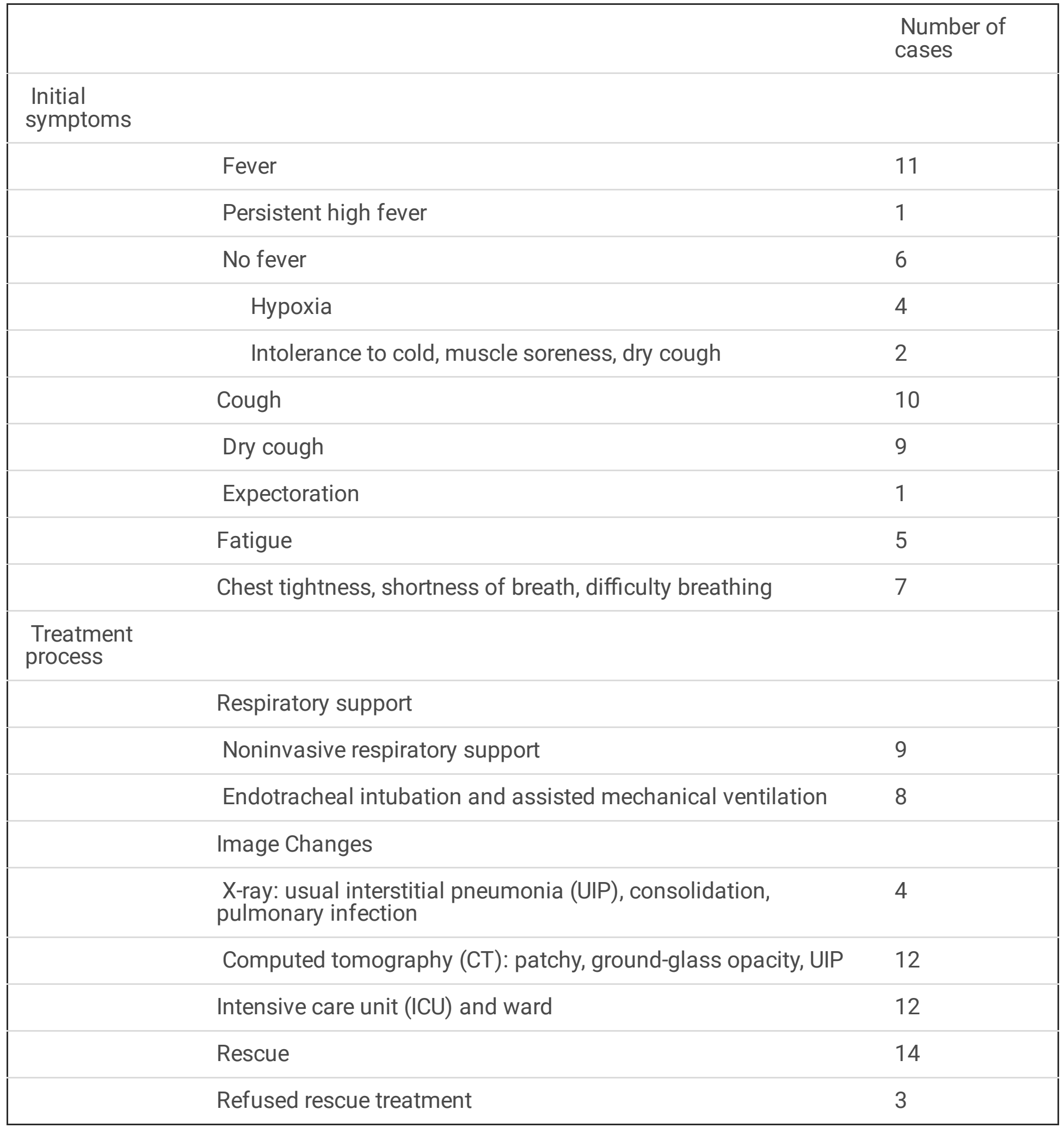

\section{Discussion:}

Human coronaviruses (HCoVs) are highly pathogenic viruses that infect the human respiratory tract. They were first isolated from the nasal secretions of patients with cold symptoms in 1965 and named B814. 
Due to the crown-like spikes on their surface under electron microscopy, they were also named HCoVs. This type of virus generally has a consistent gene structure and protein expression. In mild cases, HCoVs infect the human upper respiratory tract and the gastrointestinal tract and cause colds and diarrhea, i.e., a self-limited disease. In severe cases, HCoVs can cause bronchitis and pneumonia and even affect multiple organs, leading to death. According to phylogeny, $\mathrm{HCoV}$ s are divided into 4 subgroups, i.e., $\mathbb{\otimes}-\mathrm{CoV}$, $\beta-\mathrm{CoV}, \mathrm{Y}-\mathrm{CoV}$ and $\delta$-CoV [1]. Thus far, there are $6 \mathrm{HCoVs}$ that can infect people, and 2 have high infection rates and mortality rates. One such $\mathrm{HCoV}$ is SARS-CoV, which causes severe acute respiratory syndrome (SARS); an outbreak in 2002-03 spread to 29 countries and regions around the world, resulting in 8546 infections and 809 deaths, with a mortality rate of $9.6 \%$; however, the mortality rate in older populations is estimated to be over $50 \%$. Another $\mathrm{HCoV}$ is MERS-CoV, which causes middle east respiratory syndrome (MERS), first discovered in Saudi Arabia in 2012. At the end of 2015, it caused at least 1621 infections in 26 countries and regions around the world, resulting in 584 deaths with a mortality more than $36 \%$ [2]. After infected, the early symptoms of MERS-CoV are atypical, with subsequent severe respiratory distress and pneumonia. About one-third of patients develop gastrointestinal symptoms including vomiting and diarrhea, showing a high mortality.

2019-nCoV is an enveloped plus single-stranded RNA virus with a diameter of 60-140nm. The spikes of barrel-shaped glycoproteins in the envelope give the virus a crown-like appearance. The whole-genome sequencing of 2019-nCoV showed an $86.9 \%$ nucleotide sequence identity with the previously identified bat SARS-like coronavirus genome (bat-SL-CoVZC45, MG772933.1) [3]. The process of infection is similar to that of SARS-CoV; that is, the respiratory symptoms are severe, the gastrointestinal symptoms are mild, and the disease progresses rapidly. However, according to the public information currently obtained, it is highly contagious and can spread between people. Individuals with latent infection and no symptoms are also contagious. Person-to-person spread is thought to occur mainly via respiratory droplets and close contact. 2019-nCoV has also been found in the stool samples of patients [4,6]; however, whether 2019nCoV can spread in this way is still unclear. The Chinese population is generally susceptible to 2019$\mathrm{nCoV}$. The latent period generally ranges from 3-7 days; the longest latency period has not exceeded 14 days. Nonspecific symptoms at the early stage of infection include fever, fatigue, dry cough, sore throat, myalgia, etc. Early routine blood results indicate normal or decreased white blood cell count, reduced lymphocyte count, and, in some patients, elevated liver enzyme, muscle enzyme, and lactate dehydrogenase levels. Severe cases involve elevated serum D-dimer levels and progressively reduced peripheral blood lymphocyte counts. Nucleic acid of 2019-nCoV can be detected in throat swabs, sputum specimens, lower respiratory tract secretions, blood and fecal specimens. In the early stage, chest imaging results show multiple small plaque shadows in the peripheral lung, developing into multiple ground-glass opacities and infiltration shadows in both lungs. In severe cases, lung consolidation may occur, but pleural effusion is rare. Approximately $50 \%$ of patients develop dyspnea after 1 week, and in severe cases, the disease progresses to acute respiratory distress syndrome (ARDS) [4]. For male patients, senior patients, patients with multisystem diseases and patients with underlying pulmonary diseases, critical conditions are more prone to occur, and non-improved respiratory failure and persistent hypoxemia after treatment are associated with a high risk of death. ECMO treatment is an effective 
method to help critically ill patients [5]. Although the first patient in the United States achieved good results using remdesivir [6], its efficacy and safety still need to be verified by large-scale clinical trials. Remdesivir is currently undergoing clinical trials in a 2:1 ratio between the treatment group and the control group.

The current data show that 31774 cases have been diagnosed nationwide; 6101 were severe cases, and 27657 were suspected cases, with 722 deaths (data up to 23:59 on February 7, 2020). The mortality rate is $23.3 \%$ in China, and severe cases account for $19.2 \%$. Currently, the observed mortality for 2019 -nCoV is lower than that for SARS-CoV and MERS-CoV, but the ability to spread is stronger, with a large number of infections. The development of the outbreak cannot be underestimated, and effective isolation is still the best method to control the spread. The 17 deaths in this study indicate that the risk of death is higher in elderly patients with underlying diseases. At present, hypertension accounts for a relatively high proportion of observed underlying diseases. Studies have shown that there are 6 predictive factors for the risk of death from pneumonia: multiple lobar infiltrates, lymphocyte reduction, mixed bacterial infection, smoking history, hypertension, and age. The majority of the 17 patients were elderly and had hypertension. Hypertension is also a common underlying disease in elderly patients. Whether hypertension increases the affinity of patients to the virus and contributes to the spread of the virus remains to be revealed by further research.

In this study, only 2 of the 17 patients were tested for the 2019-nCoV pathogen, and the results were positive. However, due to the time required for virus isolation and nucleic acid sequencing and the shortage of clinical nucleic acid detection reagents, the remaining 15 patients did not undergo etiological examinations. However, in the early stage of an outbreak with limited resources, chest imaging (chest CT) can more directly aid clinical diagnosis. In this study, all the imaging results pointed to viral pneumonia, indicating that death was caused by NCIP. Additionally, due to the different development stages of disease and the varying quality of respiratory tract samples, not all patients whose etiological examination was negative can be excluded. Clinical diagnosis based on early clinical symptoms of disease combined with changes in chest imaging should implemented when resources are insufficient. Isolating older people is an effective way to reduce mortality.

\section{Conclusions:}

Among the 17 NCIP cases, the non-specific early symptoms included fever, cough, and fatigue, and the symptoms progressed to respiratory distress. All patients required tracheal intubation and assisted mechanical ventilation. Currently, there are no effective drugs for pulmonary infection, and the current treatment is mainly symptomatic supportive treatment. Some patients required pulmonary function replacement therapy. Older patients and immunocompromised patients did not show typical early symptoms, such as fever, but the disease progressed rapidly, often accompanied by persistent hypoxemia. Deaths primarily occurred in the patients over 70 years old, i.e., there were fewer deaths of patients younger than 60 years old. Males were affected more than females. Patients with underlying pulmonary diseases and multiple systemic diseases had a higher risk of death. 
Older people are at high risk of death due to 2019 -nCoV infection, and protecting high-risk groups is an effective way to reduce the number of deaths. A large number of viral pneumonia in Wuhan within a short period of time suggest the outbreak caused by a new virus, virus nucleic acid test will make accurate diagnosis, and the diagnosis based on clinical manifestations and imaging results provides a basis for early treatments.

\section{List Of Abbreviations}

ARDS: acute respiratory distress syndrome

CT: computed tomography

$\mathrm{CB}$ : chronic bronchitis

COPD: chronic obstructive pulmonary disease

CHD: coronary heart disease

ECMO: extracorporeal membrane oxygenation

HCoVs: human coronaviruses

ICU: intensive care unit

LC: liver cirrhosis

MERS: middle east respiratory syndrome

2019-nCoV: 2019 novel coronavirus

NCIP: novel coronavirus-infection pneumonia

PD: Parkinson's disease

SARS: severe acute respiratory syndrome

UIP: usual interstitial pneumonia

\section{Declarations}

\section{Ethics approval and consent to participate}

Ethical approval was obtained from Jiangsu Province Hospital of Chinese Medicine Ethics Committee.

\section{Consent for publication}


All the authors agreed to publish the research.

\section{Competing interests}

The authors declared that they have no competing financial interests.

\section{Availability of data and material}

The data and material were available publicly and could be shared.

\section{Funding}

We declare that we have no financial and personal relationships with other people or organizations that can inappropriately influence our work.

\section{Authors' contributions}

Dandan Hong and Wenyue Chen collected the data. Cheng Zhang wrote the article. Qingling Ren and Qun Guan reviewed the article and corrected it.

\section{Acknowledgements}

We thank Chao Chen who kindly assisted in arranging the manuscript and correction

\section{References}

1. Su S, Wong G, Shi W, et al. Epidemiology, Genetic Recombination, and Pathogenesis of Coronaviruses. Trends Microbiol.2016 Jun; 24(6): 490-502.

2. Manocha S, Walley KR, Russell JA. Severe acute respiratory distress syndrome (SARS): a critical care perspective. Crit Care Med.2003 Nov; 31(11): 2684-92.

3. Yushun Wan, Jian Shang, Rachel Graham, et al. Receptor recognition by novel coronavirus from Wuhan: 3 An analysis based on decade-long structural studies of SARS. J. Virol. doi: 10.1128/JVI.00127-20

4. Chen N, Zhou M, Dong X, et al. Epidemiological and clinical characteristics of 99 cases of 2019 novel coronavirus pneumonia in Wuhan, China: a descriptive study. Lancet.2020 Jan 30. pii: S01406736(20) 30211-7.

5. Mendes PV, Melro LMG, Li HY, et al. Extracorporeal membrane oxygenation for severe acute respiratory distress syndrome in adult patients: a systematic review and meta-analysis. Rev Bras Ter 
Intensiva.2019 Oct-Dec; 31(4): 548-554.

6. Holshue ML, DeBolt C, Lindquist S, et al. First Case of 2019 Novel Coronavirus in the United States. N Engl J Med.2020 Jan 31. doi: 10.1056/NEJMoa2001191.

\section{Supplementary Files}

This is a list of supplementary files associated with this preprint. Click to download.

- abstract.doc 